\title{
Importance of noise models in FSO communications
}

\author{
Muhammad N Khan
}

\begin{abstract}
Free-space optical (FSO) communication is an emerging technology which offers enormous bandwidth, license-free spectrum and highly secure link. Avalanche photodiodes (APD) are normally used for the detection of high-speed FSO signals, where the noise shows signal-dependent Gaussian noise (SDGN) distribution rather than the

signal-independent Gaussian noise (SIGN) distribution. We investigate the use of on-off keying (OOK) and low density parity check (LDPC) code on the performance of a FSO communication system. We also provide a good comparison of FSO communication noise models considering a moderate atmospheric turbulence condition. We show that large gains are possible using an LDPC decoder (i.e. at a bit error rate of $10^{-3}$, there is a gain of about $6 \mathrm{~dB}$ considering the SDGN model in case of no turbulence condition at $\lambda=10 \mathrm{~dB}$ ), when the channel state information (CSI) is known at the receiver. We develop an extrinsic information transfer (EXIT) chart to measure the decoder convergence with and without the effect of turbulence noise. It is also shown that the SDGN model should be considered for the optimum detection with significant gain of $2.5 \mathrm{~dB}$ at $\lambda=0 \mathrm{~dB}$ and about $1 \mathrm{~dB}$ at $\lambda=10 \mathrm{~dB}$.
\end{abstract}

Keywords: Signal-dependent Gaussian noise (SDGN); Signal-independent Gaussian noise (SIGN); On-off keying (OOK); Low density parity check code (LDPC); Extrinsic information transfer (EXIT) chart

\section{Introduction}

Free-space optical (FSO) communication systems are capable of providing high data transmission rates and have received considerable attention during the past few years in many applications - satellite communications, fibre backup, RF-wireless back haul and last mile connectivity [1]. In practice, the FSO communication link availability becomes limited during foggy weather and heavy snow fall [2-5]. The FSO signal intensity undergoes random fluctuation due to the atmospheric turbulence, known as scintillation. Scintillation causes performance degradation and possible loss of connectivity. These drawbacks pose the main challenge for the FSO communication system deployment. The desire to mitigate these drawbacks has generated studies of coding systems in a manner similar to their radio frequency (RF) counterparts that can improve the system performance [6,7].

Low density parity check (LDPC) code was first introduced by Gallager [7] in 1962, and its performance is nearly close to the Shannon limits. The performance of

Correspondence: dr.nasirkhan@ucp.edu.pk

Faculty of Engineering, University of Central Punjab, 1-Khayaban-e-Jinnah, Lahore 54000, Pakistan the LDPC code is better than Turbo codes [8] with iterative decoding algorithms which are easy to implement with lower decoding complexity. The LDPC code of any rate and block length can also be created easily by just changing the shape of the parity check matrix. The rate adaptability in the LDPC code can be obtained easily compared to other codes. The LDPC code has the feature of parallelism for supporting different speeds, performances and memory consumption. It is therefore better to consider the LDPC code for FSO communications because of its capacity approaching performance and comparatively easy implementation [8].

The detection of weak signals in the FSO communication is dominated by the presence of dark current due to the background noise. This problem can be overcome by using the avalanche photodiode (APD) which amplifies the electrical current due to the internal current gain [1]. The APD gain allows the reduction or elimination of noisy external amplifiers. APDs are easily available for a wide range of wavelengths. These APDs can measure even lower level light signals and are used in a wide variety of applications requiring high sensitivity [2]. APDs

\section{Springer}

(C) 2014 Khan; licensee Springer. This is an Open Access article distributed under the terms of the Creative Commons Attribution License (http://creativecommons.org/licenses/by/2.0), which permits unrestricted use, distribution, and reproduction in any medium, provided the original work is properly credited. 
are incorporated in many high-performance FSO communication applications because they enable high signalto-noise ratio (SNR). APDs are preferred over positiveintrinsic-negative (PIN) diodes due to their high internal gain characteristics and improved SNR capability [2].

In this paper, we study the signal-independent Gaussian noise (SIGN) and signal-dependent Gaussian noise (SDGN) models for the FSO communication and develop a comparison between these noise models. We also develop an error analysis along with the effects of the background noise level in both models. We performed the novel investigations of log-likelihood ratio (LLR) mappings for the SDGN and SIGN model taking into account the effect of scintillation. We are not aware of such existing coded and uncoded error analysis comparison. A decoding convergence behaviour (i.e. extrinsic information transfer (EXIT) chart) for the SDGN and SIGN models is also provided. The proposed conceptual study using the LDPC code and EXIT chart has not been introduced for such noise models.

We incorporate the implementation of APDs (i.e. considering the InGaAs APD) for the FSO communication system and present new results for the uncoded and coded bit error rate (BER) considering SDGN and SIGN models. In this paper, the communication system of interest is the same as that analysed by [9] (see references therein). However, the combined photodetection (PD) shot noise and the thermal noise of the APD has been considered as the SDGN and compared with the SIGN model. We assume both cases (i.e. with and without scintillation), and the transmitter and receiver are perfectly aligned. In our simulation, we assume that the channel state information (CSI) is known at the receiver.

The remainder of this paper is organized as follows. In Section 2, we present the system model, which provides the SDGN/SIGN model for the FSO communication system. Section 3 discusses the structure of the LDPC code used for the performance improvement of the FSO communication system under atmospheric turbulence condition. It also provides the main results of LLR mappings for the SDGN and SIGN models. The EXIT chart analysis is given in Section 4. Simulation results for the uncoded/coded BER and the EXIT chart are presented in Section 5. Section 6 provides the final concluding remarks.

\section{System model}

A system block diagram comprising the SIGN and SDGN models is shown in Figure 1. It consists of a transmitter (i.e. source, encoder and OOK mapper), channel and receiver (i.e. APD aperture, channel decoder, demapper and sink). The data bits $x \in(0,1)$ from the source encoder are encoded by the channel encoder, and the coded bits are then sent through the atmospheric turbulent channel using the OOK transmission scheme. The information bits are modulated such that the presence of light pulse transmits bit ' 1 ' and the absence transmits bit ' 0 '. We considered the atmospheric turbulence condition, background noise from the extraneous noise sources (i.e. stars and sun), and the Gaussian noise (which is considered as the SDGN and SIGN). At the receiver, the incoming optical beam is focused onto the photodetector that converts it into an electrical signal. Channel decoder decodes the received signal using the suggested coding technique and then demapped to get the estimated transmitted data.

\subsection{SDGN and SIGN models}

Following the derivation done by [10,11], we model the output of the APD photodetector as the Gaussian approximation of the Webb model. The average number of photons absorbed by an APD illuminated with total optical intensity $P$ can be expressed as

$$
\bar{n}=\left(\frac{\eta}{h_{p} v}\right) P
$$

where $h_{p}$ is Planck's constant, $v$ is the optical frequency, and $\eta$ is the quantum efficiency, which is defined as the ratio of the absorbed to the incident photons. According to [12], in an optical communication system using APD, the actual number of photons absorbed, $n$, is a Poisson distributed random variable with probability function $p(n)=\left(\bar{n}^{n} / n !\right) \exp (-\bar{n})$. The conditional probability density function (pdf) $p(k \mid \bar{n})$ of generating an avalanche of

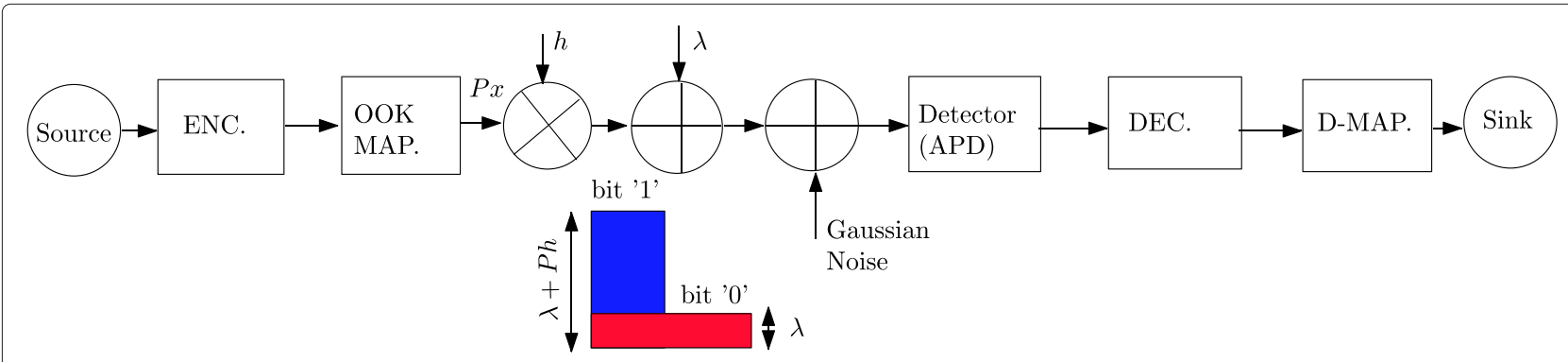

Figure 1 A typical SIGN and SDGN system model. 
$k$ output photoelectrons for the mean absorbed photons $\bar{n}$ is derived by the McIntyre-Conradi (MC) distribution [10]. The pdf obtained by MC gives the exact mathematical analysis which can be approximated by the continuous Webb density [11]. In [9], we propose the SDGN model which is the approximation of the continuous Webb density whose pdf is given by

$$
p_{\mathrm{SDGN}}(k)=\frac{1}{\sqrt{2 \pi \bar{n} G^{2} F}} \exp \left(\frac{-(k-\bar{n} G)^{2}}{2 \bar{n} G^{2} F}\right)
$$

where $F=k_{\text {eff }} G+(2-1 / G)\left(1-k_{\text {eff }}\right)$ is the excess noise factor, $G$ is the average APD gain and $k_{\text {eff }}$ is the ionization ratio constant. As shown in Figure 1, the information bit ' 1 ' is represented by $\bar{n}_{1}$ (i.e. assuming $\eta=1, \bar{n}_{1}=\lambda+P h$, which is considered to be the background level $\lambda$ plus the signal intensity $P$ and $h$ shows the effect of scintillation) and bit ' 0 ' by $\bar{n}_{0}$ (i.e. $\bar{n}_{0}=\lambda$ ) [13]. For intensity fluctuations, we adopt the log-normal (LN) distribution that best describes the moderate turbulence conditions [14]. In [14], irradiance fluctuations for the moderate scintillation were shown to be well modelled by a LN distribution, and the pdf of the LN distribution is given by

$$
p_{h}(h)=\frac{1}{\sqrt{2 \pi \sigma_{\operatorname{lnh}}^{2}} h} \exp \left[-\frac{(\log h-\mu \operatorname{lnh})^{2}}{2 \sigma_{\ln h}^{2}}\right]
$$

where $\mu_{\ln h}$ and $\sigma_{\ln h}^{2}$ are the mean and variance of the logarithm of $h$. It is assumed that $E[h]=1$ so that the average received optical power remains constant, and from the moments of the LN distribution, it follows that $\mu_{\ln h}=$ $-\frac{1}{2} \sigma_{\ln h}^{2}$ and $\sigma_{\operatorname{lnh}}^{2}=\log \left(1+\sigma_{I}^{2}\right)$, where $\sigma_{I}^{2}$ is the scintillation index (SI) defined in [15]. In [9], we relate physical parameters of the APD with statistical parameters of the SDGN model by saying $\mu_{x}=\bar{n}_{x} G$ and $\sigma_{x}^{2}=\bar{n}_{x} G^{2} F$. The relationship shows the dependence of statistical parameters (i.e. $\mu_{x}$ and $\sigma_{x}^{2}$ ) on physical parameters (i.e. $G$ and $F$ ) of the APD.

In [9], we exploit the concept of the double Gaussian noise model [13], with the name of SDGN model and did the investigation for the optimum/sub-optimum detectors $^{\mathrm{a}}$. In this paper, we exploit the concept of the SDGN model and compare its performance with the SIGN model. We also implement the LDPC decoder to improve the system performance under turbulent conditions. For the SIGN model, the variance $\left(\sigma^{2}\right)$ does not depend on the signal intensity, and its pdf is derived as

$$
p_{\mathrm{SIGN}}(k)=\frac{1}{\sqrt{2 \pi \sigma^{2}}} \exp \left(\frac{-(k-\bar{n} G)^{2}}{2 \sigma^{2}}\right) .
$$

For an APD detector, parameters used are the APD internal current gain $G, k_{\text {eff }}$ and quantum efficiency $\eta$. We relate physical parameters of the APD with our signal model for the Gaussian approximation: $\mu_{0} \approx \lambda G, \mu_{1} \approx$ $(\lambda+P h) G, \sigma_{0}^{2} \approx \lambda G^{2} F, \sigma_{1}^{2} \approx(\lambda+P h) G^{2} F[9]$. In [9], we simulate density functions for the Webb and SDGN model considering the InGaAs APD with $G=10, k_{\text {eff }}=0.45$ and $F=5.5$ [16]. Simulation results in [9] show the agreement between the Webb and SDGN model near peaks of distributions. These results illustrate the simulation results of the effect of varying background level for the SDGN and SIGN models which are referred to in Section 5.

\subsection{Channel model}

The received signal for the SDGN and SIGN models after optical/electrical conversion can be given by (5) and (6), respectively.

$$
\begin{aligned}
& y_{\text {SDGN }}=\left\{\begin{array}{lr}
\mathcal{N}\left(\mu_{1}, \sigma_{1}^{2}\right) & \text { Signal presence } \\
\mathcal{N}\left(\mu_{0}, \sigma_{0}^{2}\right) & \text { Signal absence }
\end{array}\right. \\
& y_{\text {SIGN }}=\left\{\begin{array}{lr}
\mathcal{N}\left(\mu_{1}, \sigma^{2}\right) & \text { Signal presence } \\
\mathcal{N}\left(\mu_{0}, \sigma^{2}\right) & \text { Signal absence }
\end{array}\right.
\end{aligned}
$$

The FSO channel, LLR mappings for the SIGN and SDGN can be calculated as

$$
\Lambda=\log \left(\frac{p(x=0 \mid y, h)}{p(x=1 \mid y, h)}\right)
$$

where $p(x=1 \mid y, h)$ represents the probability of $x=$ 1 given the received symbol $y$ (i.e. either the SIGN and SDGN) under a certain channel condition and $p(x=0 \mid y, h)$ represents the probability of $x=0$ given the received symbol $y$ (i.e. either the SIGN and SDGN) under a certain channel condition. By the application of Bayes rule and assuming equi-likely input bits, (7) is replaced by

$$
\Lambda=\log \left(\frac{p(y \mid x=0, h)}{p(y \mid x=1, h)}\right) .
$$

For the not equi-likely input bits (i.e. $p(x) \neq 1 / 2$ ), we can rewrite (8) as

$$
\Lambda=\log \frac{p(y \mid x=0, h)}{p(y \mid x=1, h)}+L(x)
$$

where $L(x)=\log \left(\frac{p(x=0)}{p(x=1)}\right)$. The LLR mappings are analysed so that we can use the LLR mappings for the calculation of the uncoded and coded BER.

\section{Low density parity check code}

The simple operation of the low density parity check code (LDPC) code is explained by [7]. In this paper, we adopt the same approach described by [8] and develop an LDPC code by designing parity check matrices that were sparsely populated with non-zero elements using progressive edge growth (PEG) software [17]. We develop the $(3,6)$-regular half-rate code, whose graphical representation is shown by the Tanner graph [18] in Figure 2. It consists of two sets of nodes: variable nodes and check nodes. The number of edges that join variable nodes and check nodes is equal to the number of $1 \mathrm{~s}$ in the parity check matrix. The decoding of the LDPC code is performed by a well-known 


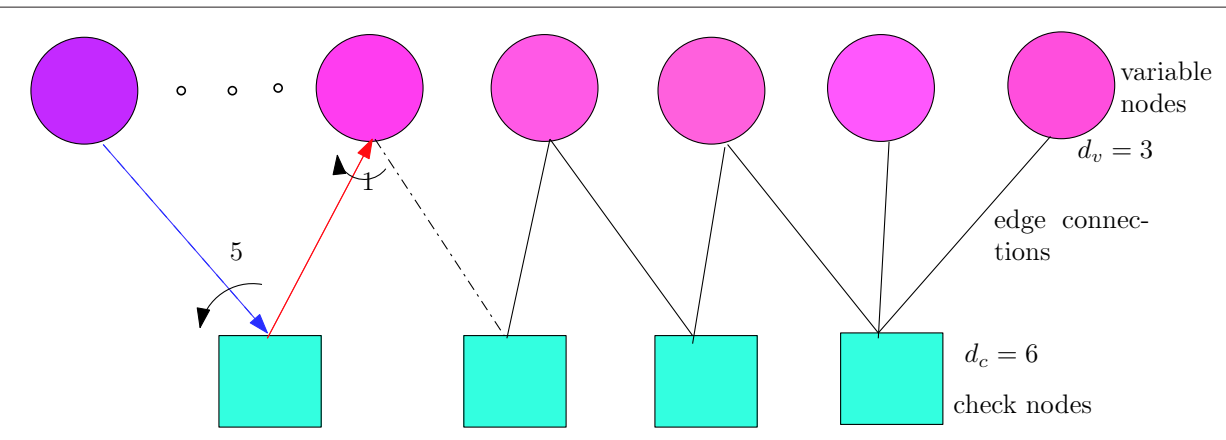

Figure 2 Tanner graph of (3,6)-regular half-rate LDPC code.

class of algorithms collectively known as message-passing algorithms (MPA) (i.e. sum-product decoding algorithms (SPA)) [8]. The operation of SPA can be explained by passing of messages (mutual information in our analysis referred to in Section 4) along the edges of the Tanner graph. The SPA is based on probabilities of received bits, which represent a level of confidence about codeword bits.

\subsection{Uncoded BER}

Considering a typical FSO communication system, the information signal from the laser is directed towards the optical receiver along the line-of-sight path. At the receiver, we perform soft demodulation of the received signal, which is to be considered in the form of LLR mappings. We consider the signal presence or absence according to the detection threshold, which we derived in [9] for the SDGN model. For the SIGN model, we consider the decision threshold midway between the mean of the two signal distributions.

To begin, we derive the LLR expression for the SDGN and SIGN models using the OOK modulation scheme. In order to calculate the uncoded BER of the FSO communication system shown in Figure 1, we need to make some assumptions for the SDGN model ${ }^{\mathrm{b}}$. In [9], we assume a simplistic Poisson approximation in the derivation of LLR mappings for the optimum detector. On the basis of our defined model, we define the maximum likelihood decision (MLD) rule which maximizes the probability of a given sequence of observations corresponding to some threshold value for OOK.

\subsubsection{SDGN LLR mapping}

For the SDGN model, channel conditional probabilities for bit ' 0 ' and ' 1 ', i.e. $p\left(y_{\mathrm{SDGN}} \mid x=0\right) \sim \mathcal{N}\left(\mu_{0}, \sigma_{0}^{2}\right)$ and $p\left(y_{\mathrm{SDGN}} \mid x=1\right) \sim \mathcal{N}\left(\mu_{1}, \sigma_{1}^{2}\right)$, are given by

$$
p\left(y_{\mathrm{SDGN}} \mid x=0\right)=\frac{1}{\sqrt{2 \pi \sigma_{0}^{2}}} \exp \left(-\frac{\left(y_{\mathrm{SDGN}}-\mu_{0}\right)^{2}}{2 \sigma_{0}^{2}}\right)
$$

$$
p\left(y_{\mathrm{SDGN}} \mid x=1\right)=\frac{1}{\sqrt{2 \pi \sigma_{1}^{2}}} \exp \left(-\frac{\left(y_{\mathrm{SDGN}}-\mu_{1}\right)^{2}}{2 \sigma_{1}^{2}}\right) .
$$

By using (10) and (11) in (8), we get OOK LLR mappings for the SDGN $\left(\Lambda_{\mathrm{SDGN}}\right)$ assuming equi-likely input bits and with no atmospheric turbulence effect,

$$
\begin{aligned}
\Lambda_{\mathrm{SDGN}}= & \frac{1}{2} \log \left(1+\frac{P}{\lambda}\right)+\frac{1}{2 G^{2} F \lambda\left(1+\frac{\lambda}{P}\right)} \\
& \times\left[\left(G \lambda-y_{\mathrm{SDGN}}\right)\left(G \lambda+y_{\mathrm{SDGN}}\right)+G^{2} \lambda P\right] .
\end{aligned}
$$

Similarly, we derived LLR mappings considering the turbulence noise case as

$$
\begin{aligned}
\Lambda_{\mathrm{SDGN}}= & \frac{1}{2} \log \left(1+\frac{P h}{\lambda}\right)+\frac{1}{2 G^{2} F \lambda\left(1+\frac{\lambda}{P h}\right)} \\
& \times\left[\left(G \lambda-y_{\mathrm{SDGN}}\right)\left(G \lambda+y_{\mathrm{SDGN}}\right)+G^{2} \lambda P h\right] .
\end{aligned}
$$

\subsubsection{SIGN LLR mapping}

For the SIGN model, channel parameters are related as, i.e. $\mu_{0}=\lambda G, \mu_{1}=(\lambda+P h) G, \sigma^{2}=(\lambda+0.5 P h) G^{2} F$. The variance here is considered to be the average value for the background noise and signal level. For bit ' 1 ', we receive the signal and the background levels at the APD detector, whereas for bit ' 0 ', only the background level is received. The channel conditional probabilities for bits ' 0 ' and ' 1 ' in the case of the SIGN model, i.e. $p\left(y_{\text {SIGN }} \mid x=\right.$ $0) \sim \mathcal{N}\left(\mu_{0}, \sigma^{2}\right)$ and $p\left(y_{\text {SIGN }} \mid x=1\right) \sim \mathcal{N}\left(\mu_{1}, \sigma^{2}\right)$, are

$$
p\left(y_{\mathrm{SIGN}} \mid x=0\right)=\frac{1}{\sqrt{2 \pi \sigma^{2}}} \exp \left(-\frac{\left(y_{\mathrm{SIGN}}-\mu_{0}\right)^{2}}{2 \sigma^{2}}\right)
$$

$$
p\left(y_{\mathrm{SIGN}} \mid x=1\right)=\frac{1}{\sqrt{2 \pi \sigma^{2}}} \exp \left(-\frac{\left(y_{\mathrm{SIGN}}-\mu_{1}\right)^{2}}{2 \sigma^{2}}\right) .
$$


By using (14) and (15) in (8), we get LLR mappings for the SIGN $\left(\Lambda_{\text {SIGN }}\right)$ assuming equi-likely input bits and no scintillation case,

$$
\Lambda_{\mathrm{SIGN}}=\frac{G P}{2 \sigma^{2}}\left[G(2 \lambda+P)-2 y_{\mathrm{SIGN}}\right] .
$$

Similarly, considering the scintillation, we derive LLR mappings as

$$
\Lambda_{\mathrm{SIGN}}=\frac{G P h}{2 \sigma^{2}}\left[G(2 \lambda+P h)-2 y_{\mathrm{SIGN}}\right]
$$

\subsection{Coded BER}

Once we derived the soft values, i.e. LLR mappings for the SDGN and SIGN model in subsections 3.1.1 and 3.1.2, we pass those values to the decoder to decode the message and evaluate the probability of bit error. For the decoding purpose, we use the same SPA algorithm proposed in $[7,8]$.

\section{SDGN/SIGN EXIT curves}

The EXIT chart is used to predict the convergence behaviour of the iterative decoder. It is based only on the simulated behaviour of the individual component decoder (i.e. variable and check nodes) to obtain the desired transfer characteristics [19]. It tracks the mutual information (MI) of extrinsic LLRs (i.e. $L_{v}$ shown in Figure 3) rather than their densities at each iteration and gives an excellent visual representation of the decoder. ten Brink proposed EXIT chart characteristics based on MI in [6], which shows the flow of extrinsic information through the soft in/soft out decoders. Hagenauer described the EXIT chart in iterative processing in [19]. In this paper, we develop EXIT curves in order to measure the performance comparison of the decoder for both the SIGN and SDGN models. The EXIT chart curves consist of three main curves: variable node curve, check node curve and the trajectory of the decoder convergence.
In the FSO communication system, an information sequence is encoded into a bit stream considering the FSO channel symbol. Then, the received signals are demapped as shown in Figure 3. Demapping is an important step before soft decision decoding. The FSO demapper needs to be designed to demap the received signals. The LLR demapping algorithm proposed in [20] works well in traditional uniform modulations, but they result in channel capacity loss. Therefore, the iterative demapping algorithm derived by ten Brink in [21] with a priori knowledge of other bits corresponding to the same bits was employed in many systems [22].

It is suggested in [6] that the a priori input (i.e. $L_{A}$ shown in Figure 3) to the constituent decoder can be modelled as an independent Gaussian random variable $z_{A}$ with mean 0 and variance $\left(\sigma_{A}^{2}\right)$ in conjunction with the unmapped data bits $s \in(0,1)$.

$$
L_{A}=\mu_{A}(1-2 s)+z_{A}
$$

Since $L_{A}$ is supposed to be an LLR mapping based on the simple Gaussian distribution, in the case of satisfying the symmetric and consistency condition for the a priori channel, the mean value $\left(\mu_{A}\right)$ for the a priori channel must fulfil [19]

$$
\mu_{A}=\sigma_{A}^{2} / 2
$$

According to [6], to measure the MI of the a priori knowledge, the $I_{A V}(A ; S)$ between the equi-likely inputs and the respective LLR can be simplified as

$$
\begin{aligned}
I_{A V}(A ; S)= & \frac{1}{2} \sum_{s \in(0,1)} \int_{-\infty}^{-\infty} p_{A}(\xi \mid S=s) \log _{2} \\
& \times\left(\frac{2 p_{A}(\xi \mid S=s)}{p_{N}(\xi \mid S=0)+p_{N}(\xi \mid S=1)}\right) d \xi
\end{aligned}
$$

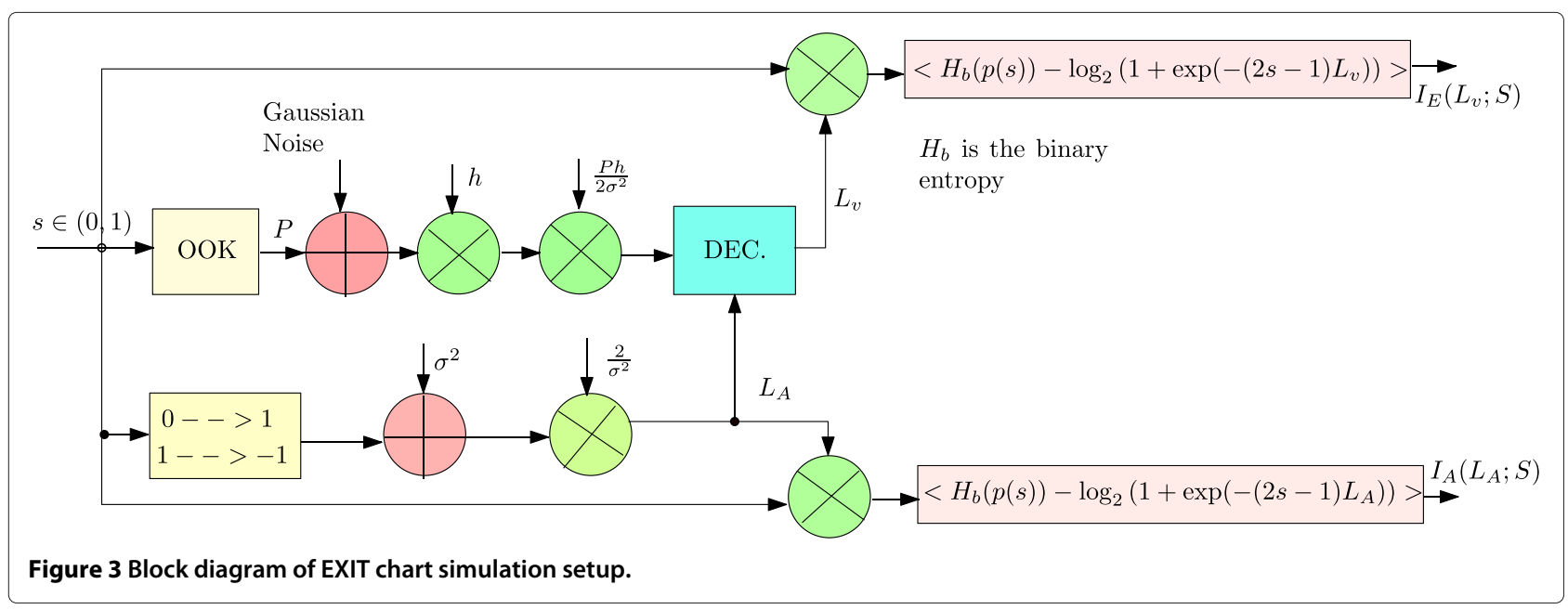


where $\mathrm{p}_{\mathrm{A}}(\xi \mid \mathrm{S}=\mathrm{s})=\frac{1}{\sqrt{\left(2 \pi \sigma_{\mathrm{A}}^{2}\right)}} \exp \left(\frac{\left(\xi-\left(\sigma_{\mathrm{A}}^{2} / 2\right) \mathrm{s}\right)^{2}}{2 \sigma_{\mathrm{A}}^{2}}\right)$. For the equip-likely input bits, (20) can be further simplified to as

$$
I_{A V}(A ; S)=1-\mathcal{E}\left\{\log _{2}\left(1+\exp \left(-(2 s-1) L_{A}\right)\right)\right\}
$$

where $\mathcal{E}$ is the expectation operator. The MI is also used to quantify the extrinsic output $I_{E V}\left(L_{v} ; S\right)$ as

$$
I_{E V}\left(L_{v} ; S\right)=1-\mathcal{E}\left\{\log _{2}\left(1+\exp \left(-(2 s-1) L_{v}\right)\right)\right\}
$$

where $L_{v}$ denotes the channel LLR (i.e. either the $\Lambda_{\mathrm{SDGN}}$ and/or $\left.\Lambda_{\text {SIGN }}\right)$. The main components of the EXIT chart curves are the EXIT functions of the component decoder, which relates the a priori $\mathrm{MI}\left(I_{A V}, I_{A C}\right)$ at the input and the extrinsic MI $\left(I_{E V}, I_{E C}\right)$ at the output of component decoder as we described in [5]. We can measure the extrinsic MI $\left(I_{E C}\right)$ of the check node decoder using (6) in [5].

\section{Simulation results}

To analyse the BER performance of the SDGN and SIGN models for the OOK, we conduct a number of Monte Carlo simulations. For the SDGN and SIGN detection, each trial involved generating a block of random OOK symbols. We are computing the respective LLRs for the OOK, making soft decisions and finally counting the number of bit errors. These results are performed with and without considering the effect of scintillation. The signalto-noise ratio (SNR) is calculated on the basis of $\frac{\mu_{1}-\mu_{0}}{\sigma_{0}^{2}}=$ $\frac{P}{\lambda}$.

In Figure 4, we show simulation results for the OOK without scintillation effect case for the SDGN and SIGN models using (12) and (16), respectively. The uncoded BER of the SDGN and SIGN detection for different background irradiation levels is plotted. We derived the detection threshold for the SDGN model in [9], and the detection threshold for the SIGN model is considered the midway of the two signal distributions. It is reported in [1] that the InGaAs APD is a good detector for the $\mathrm{GHz}$ photon detection at $1,550 \mathrm{~nm}$. We consider the InGaAs APD with physical parameters $G=10, k_{\text {eff }}=0.45$ [16], and the BER was determined as a function of the SNR for $\lambda=0$ and $10 \mathrm{~dB}$.

We present simulation results for the background level of 0 and $10 \mathrm{~dB}$ because we want to compare the performance of the APD detector for a very low to moderate background level. We developed such sort of analysis for various values of background level in [9] considering the PIN photodiode. We measure the effect of background level on the system performance and provide a good insight for the evaluation of SDGN and SIGN models. After looking into the simulation results for low and moderate background levels, we can see that the SDGN model can be approximated by SIGN at high background levels (i.e. the difference in performance in terms of BER

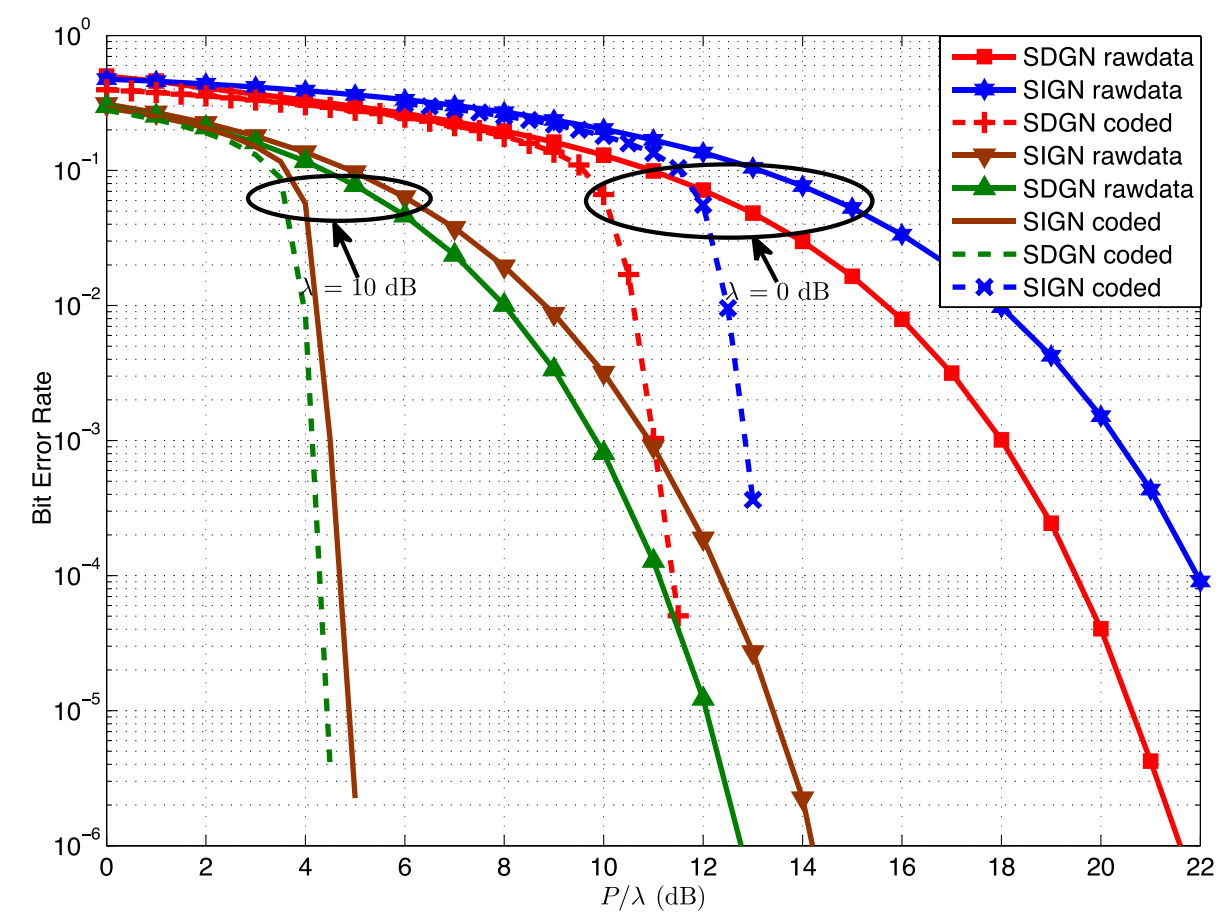

Figure 4 Coded and uncoded BER of SDGN and SIGN models without the effect of scintillation. 
Table 1 Comparison of SDGN/SIGN models at a BER of $10^{-3}$ for the uncoded system

\begin{tabular}{|c|c|c|c|c|c|c|}
\hline & & & Values & & & \\
\hline \multicolumn{7}{|c|}{ Without atmospheric turbulence effect $(S I=0)$} \\
\hline$\lambda(\mathrm{dB})$ & 0 & 2 & 4 & 6 & 8 & 10 \\
\hline SIGN model SNR (dB) & 20.50 & 18.50 & 16.50 & 14.56 & 12.75 & 11.0 \\
\hline SDGN model SNR (dB) & 18.0 & 16.25 & 14.50 & 13.0 & 11.45 & 10.0 \\
\hline Gain (dB) & 2.5 & 2.25 & 2.0 & 1.56 & 1.35 & 1.0 \\
\hline \multicolumn{7}{|c|}{ With atmospheric turbulence effect $(S I=1)$} \\
\hline$\lambda(\mathrm{dB})$ & 0 & 2 & 4 & 6 & 8 & 10 \\
\hline SIGN model SNR (dB) & 30.0 & 28.25 & 26.15 & 24.50 & 22.15 & 20.0 \\
\hline SDGN model SNR (dB) & 24.0 & 22.75 & 21.15 & 20.50 & 18.75 & 17.0 \\
\hline Gain (dB) & 6.0 & 5.50 & 5.0 & 4.0 & 3.40 & 3.0 \\
\hline
\end{tabular}

between the SIGN and SDGN models is decreasing by increasing the background level, which is $1 \mathrm{~dB}$ at BER = $10^{-3}$ for $\lambda=10 \mathrm{~dB}$ ). It is also noted that the SDGN model performs better compared to the SIGN model in terms of improving SNR under low background levels, which is $2.5 \mathrm{~dB}$ at $\mathrm{BER}=10^{-3}$ for $\lambda=0 \mathrm{~dB}$.

The simulation result shows that if the background level $\lambda$ increases (e.g. from $\lambda=0$ to $10 \mathrm{~dB}$ ), the difference between the SDGN and SIGN BER curves decreases. This agrees with [9] that for a large number of background levels, the SDGN model can be approximated by a simple SIGN model. We further analyse the BER results for varying the background level from 0 to $10 \mathrm{~dB}$ and provide the results in Table 1. In Table 1, we perform simulation with and without considering the effect of scintillation. It can be seen that using the SDGN model, we can get a gain of $6 \mathrm{~dB}$ at $\lambda=0 \mathrm{~dB}$ and $3 \mathrm{~dB}$ at $\lambda=10 \mathrm{~dB}$ under the scintillation effect, whereas we can get $2.5 \mathrm{~dB}$ to $1 \mathrm{~dB}$ without scintillation effect at a BER of $10^{-3}$.

In Figure 5, we present simulation results considering the effect of scintillation where we consider the LN distribution with SI = 1 using (13) and (17). We generate $(3,6)$-regular half-rate code using the PEG algorithm [17] with $K=1,000$ and $N=2,000$. We use the standard SPA [8] to decode the received noisy symbol with 50 iterations and $10^{4}$ simulation runs. It is noted in Figures 4 and 5 that

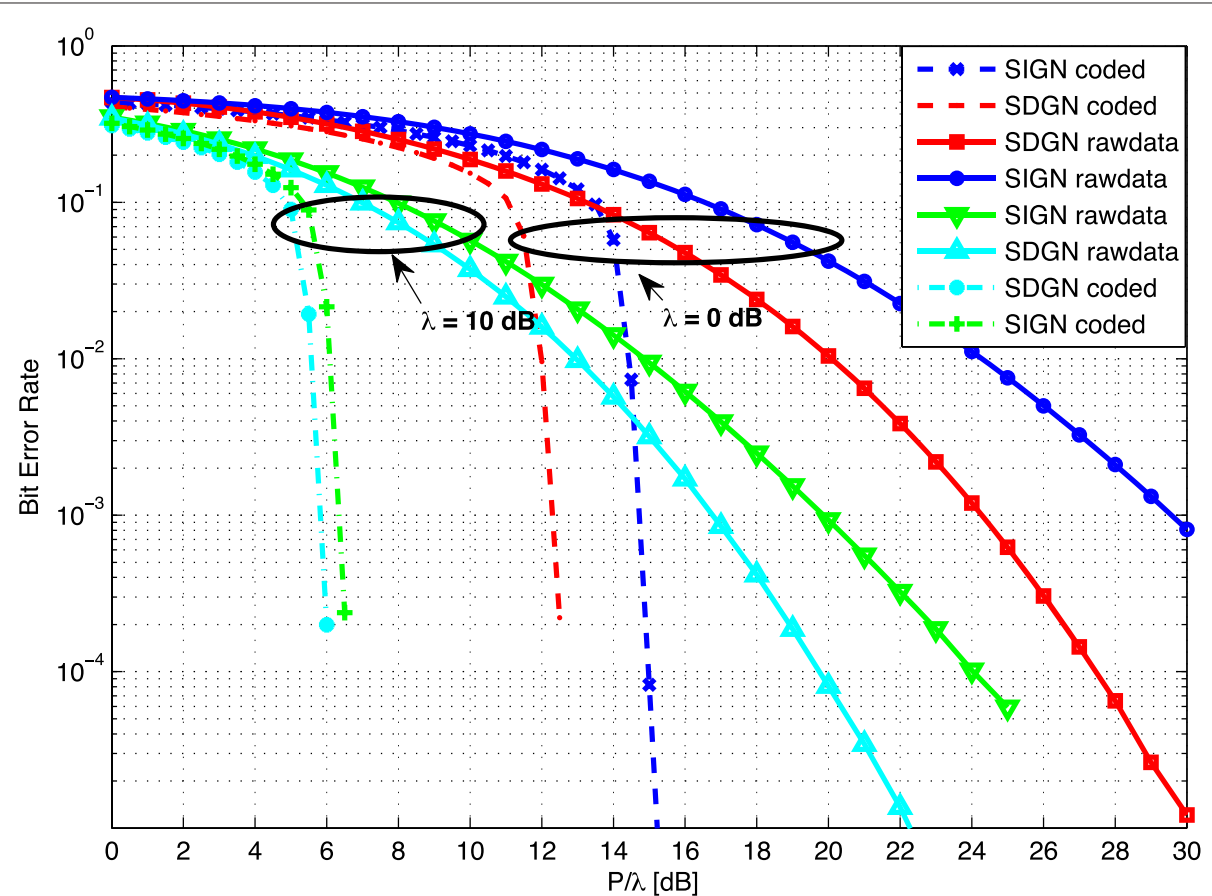

Figure 5 Coded and uncoded BER of SDGN and SIGN models considering effect of scintillation with scintillation index $=1$. 


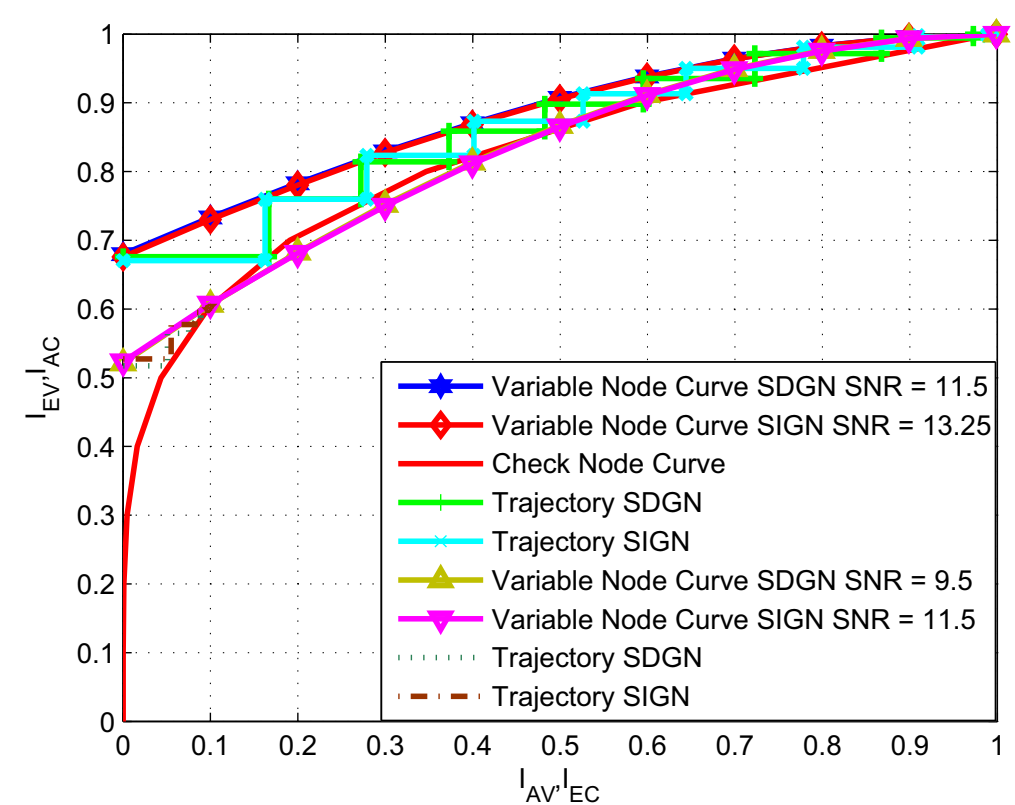

Figure 6 Extrinsic MI curves of the variable and check node with decoder trajectory for SDGN and SIGN at $\lambda=0 \mathrm{~dB}$. Without scintillation effect; SNR is in decibels (dB).

the performance of the system is improved by using the LDPC code with less optical power considering the $\lambda=0$ and $10 \mathrm{~dB}$. It is seen from the simulation results that we can achieve better performance gain for $\lambda=0$ and $10 \mathrm{~dB}$ for both values of SI (i.e. 0 and 1 ). It shows a good improvement of up to 10 to $12 \mathrm{~dB}$ coding gain at a $\mathrm{BER}=10^{-3}$ for $\lambda=0$ to $10 \mathrm{~dB}$.
In Figure 6, we show the simulation results to measure the decoder convergence behaviour for $\lambda=0 \mathrm{~dB}$. The decoder convergence measures the decoding capability of the decoder. In the simulation, we measure the MI of the variable and check node curves and the decoder trajectory after each iteration. We measure the variable node extrinsic curve using (22) and check node curve

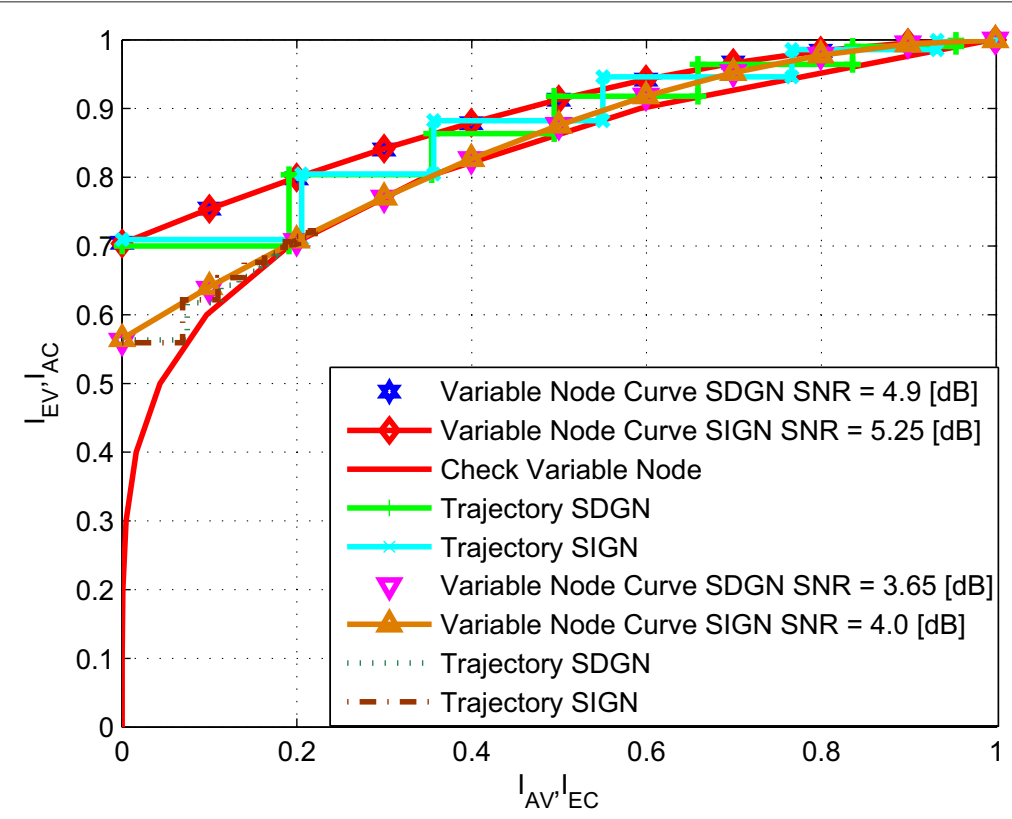

Figure 7 Extrinsic MI curves of the variable and check node with decoder trajectory for SDGN and SIGN at $\lambda=10 \mathrm{~dB}$. Without scintillation effect. 


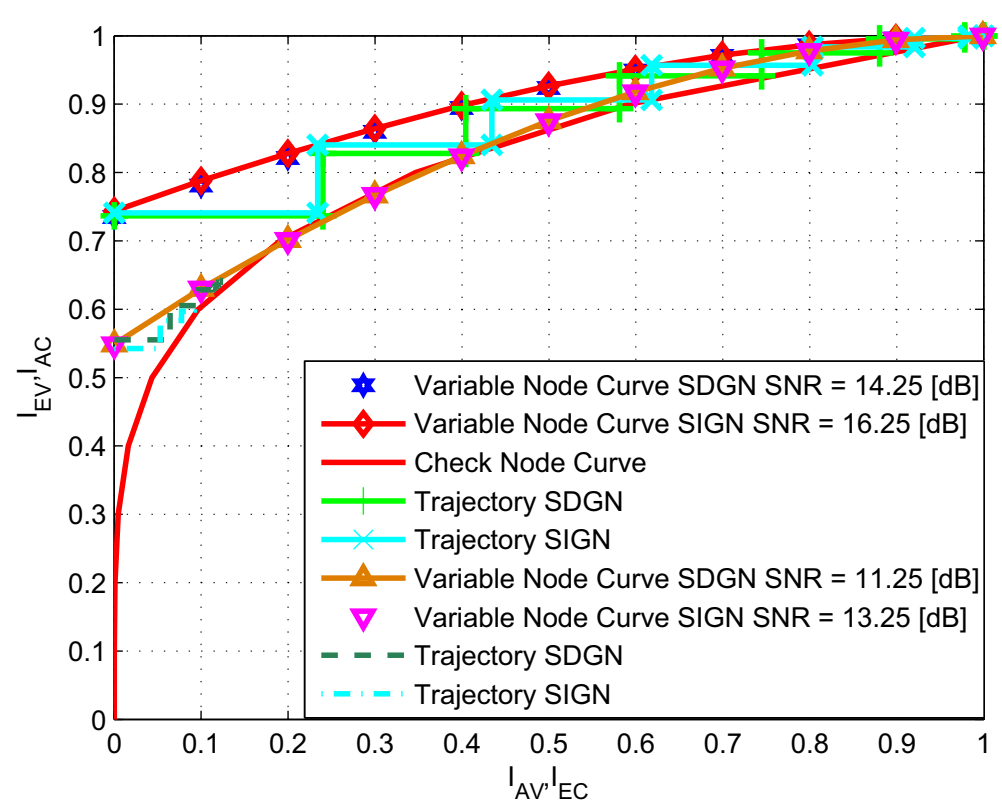

Figure 8 Extrinsic MI curves of the variable and check node with decoder trajectory for SDGN and SIGN at $\lambda=0 \mathrm{~dB}$. With scintillation effect at $S I=1$.

using (6) in [5]. It is noted that the tunnel closes at $\mathrm{SNR}=9.5 \mathrm{~dB}$ for the SDGN and at SNR $=11.5 \mathrm{~dB}$ for the SIGN, and the decoder does not converge. In this case, the decoder fails to decode the received signal. However, at $\mathrm{SNR}=11.5 \mathrm{~dB}$ for the SDGN and $13.25 \mathrm{~dB}$ for the SIGN, the tunnel opens and the decoder converges completely. It also confirms our BER simulation results obtained in Figure 4. Similarly for $\lambda=10 \mathrm{~dB}$, we show the simulation results in Figure 7. It also shows the same behaviour of convergence of the decoder for the SIGN and SDGN models. It is noted that for low background levels, the difference of SNR between the SDGN and SIGN is greater than at high background levels. It seems that for high background level, we can use the SIGN model, but

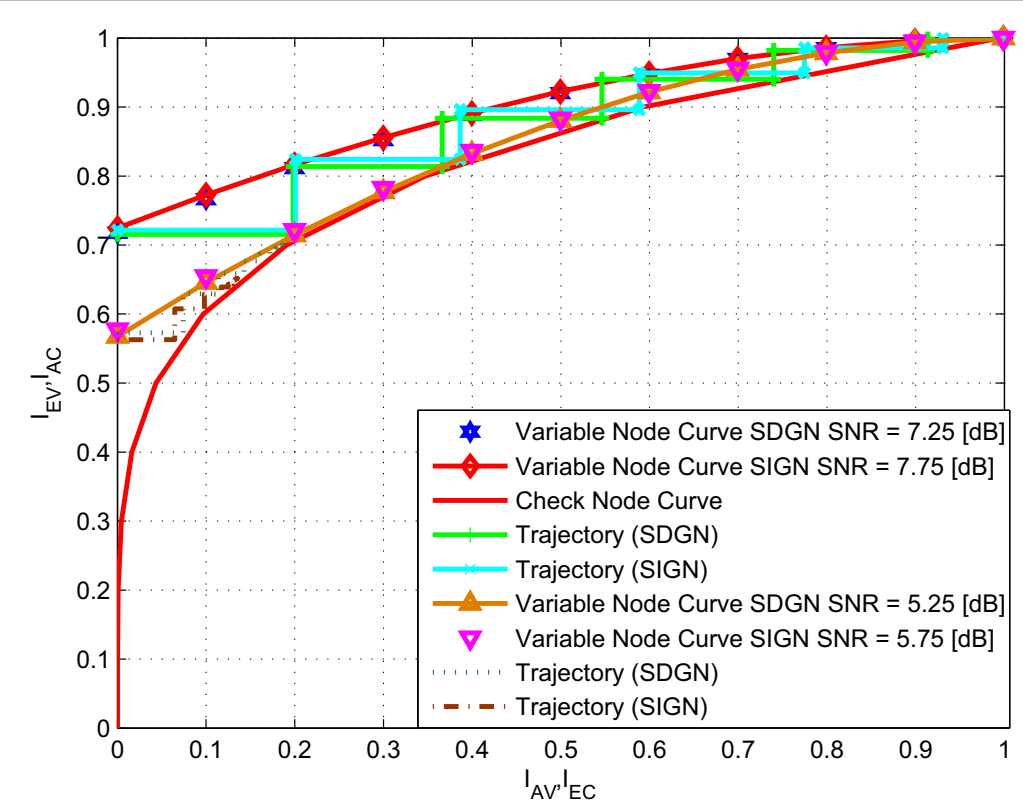

Figure 9 Extrinsic MI curves of the variable and check node with decoder trajectory for SDGN and SIGN at $\lambda=10 \mathrm{~dB}$. With scintillation effect at SI $=1$. 
for the low background level, the SDGN model should be considered.

In Figure 8, we show the simulation results to measure the decoder convergence behaviour for $\lambda=0 \mathrm{~dB}$ under the effect of scintillation. We perform the simulation for both SIGN and SDGN models. Again, we measure the corresponding extrinsic MI and the decoder trajectory in the same manner as we measure above. It is noted that the tunnel closes at $\mathrm{SNR}=11.25 \mathrm{~dB}$ for the SDGN and at $\mathrm{SNR}=13.25 \mathrm{~dB}$ for the SIGN. It means that the decoder does not converge and the decoding is a failure. However, at $\mathrm{SNR}=14.25 \mathrm{~dB}$ for the SDGN and $16.25 \mathrm{~dB}$ for the SIGN, the tunnel opens and the decoder converges completely, which means that the decoding is successful. It also confirms our BER simulation results obtained in Figure 5. Similarly for $\lambda=10 \mathrm{~dB}$, we show the simulation results in Figure 9 and get the same behaviour as can be seen from the previous results. We can conclude that the code design for the SDGN models will be optimal for the SIGN models since the shape of the variable node curve is quite the same. The waterfall region of the decoding results shown in Figures 5 and 4 can be compared with the EXIT chart simulation and found the best agreement of the decoder convergence for all scenarios.

\section{Conclusions}

We analyse the uncoded and coded BER for the proposed SDGN and SIGN models and investigate the performance of the LDPC code considering the OOK modulation scheme. New results for LLR mappings have been derived for both the SDGN and SIGN models. It is seen from the simulation results that we can get better performance for the SDGN than the SIGN model under low background levels. The performance of the SDGN model can further be increased by coding gains using the LDPC decoder. We can conclude that for a large number of background levels, the SDGN model can be approximated by the SIGN model, but at low background levels, the SDGN model should be used. The proposed research work can be extended for the hybrid FSO/RF communication under the consideration of different channel models.

\section{Endnotes}

${ }^{a}$ We say the optimum detection when the noise variance is dependent on the input bits, and sub-optimum when the noise variance is independent of the input bits.

${ }^{b}$ Variance in the signal slot is greater than the variance in the non-signal slot $\sigma_{1}^{2}>\sigma_{0}^{2}$.

\section{Competing interests}

The authors declare that they have no competing interests.

\section{Acknowledgements}

The author would like to thank Prof. Bill Cowley and Dr. Khoa D. Nguyen from the Institute for Telecommunication Research, South Australia for the thorough discussion and help in fulfilling this task and Dr. N. Letzepis from
DSTO for providing helpful support and useful suggestions during the course of investigation.

Received: 30 January 2014 Accepted: 26 May 2014

Published: 19 June 2014

\section{References}

1. SB Alexander, Optical Communication Receiver Design. (SPIE-International Society for Optical Engineering, USA, 1997)

2. RM Gagliardi, S Karp, Optical Communications. (Wiley, New York, 2006)

3. M ljaz, Z Ghassemlooy, H Le-Minh, S Rajbhandari, J Perez, A Gholami, Bit error rate measurement of free space optical communication links under laboratory-controlled fog conditions, in 16th European Conference on Networks and Optical Communications (NOC) (Newcastle-Upon-Tyne, 2011), pp. 52-55

4. MN Khan, WG Cowley, Nguyen K D, Link adaptation of FAHOR communication system, in Australian Communications Theory Workshop (AusCTW'12) (Wellington, New Zealand, 2012), pp. 120-125

5. MN Khan, WG Cowley, KD Nguyen, Puncturing optimization algorithm and its applications in free space communications, in Australian Communications Theory Workshop (AusCTW'13) (Adelaide, Australia, 2013), pp. 152-157

6. S ten Brink, Designing iterative decoding schemes with the extrinsic information transfer chart. AEU Int. J. Electron. Commun. 54(6), 389-398 (2000)

7. R Gallager, Low-density parity-check codes. Inf. Theory, IRE Trans. on. 8(1), 21-28 (1962)

8. S Johnson, Iterative Error Correction: Turbo, Low-Density Parity-Check and Repeat-Accumulate Codes. (Cambridge University Press, New York, 2010)

9. MN Khan, WG Cowley, Signal dependent Gaussian noise model for FSO communications, in Australian Communications Theory Workshop (AusCTW'11) (Melbourne, Australia, 2011), pp. 142-147

10. RJ Mclntyre, The distribution of gains in uniformly multiplying avalanche photodiodes: theory. IEEE Trans. Electron. Devices. 19(6), 703-713 (1972)

11. P Webb, Intyre McR, J Conradi, Properties of avalanche photodiodes. RCA Rev. 35, 234-278 (1974)

12. M Srinivasan, $V$ Vilnrotter, Performance of the optimum receiver for pulse-position modulation signals with avalanche photodiode statistics. Telecommunications Mission Operations Progress Report 42. 133 (2000)

13. S Dolinar, D Divsalar, J Hamkins, F Pollara, Capacity of pulse-position modulation (PPM) on Gaussian and Webb channels. Telecommunications Mission Operations Progress Report 42-142, 1-31 (2000)

14. N Letzepis, I Holland, W Cowley, The Gaussian free space optical MIMO channel with Q-ary pulse position modulation. IEEE Trans. on Wireless Commun. 7(5), 1744-1753 (2008)

15. LC Andrews, RL Phillips, Laser Beam Propagation Through Random Media. (Society of Photo Optical, SPIE, Press, Bellingham, Washington, USA, 2005)

16. P Elmer, Avalanche Photodiodes: A User's Guide. (Technical information PerkinElmer Optoelectronics, PerkinElmer, Fremont, CA, 2006)

17. X-Y Hu, E Eleftheriou, DM Arnold, Regular and irregular progressive edge-growth tanner graphs. IEEE Trans. on Inf. Theory. 51(1), 386-398 (2005)

18. R Tanner, A recursive approach to low complexity codes. IEEE Trans. on Inf. Theory. 27(5), 533-547 (1981)

19. J Hagenauer, The EXIT chart-introduction to extrinsic information transfer in iterative processing, in Proceeding 12th European Signal Processing Conference (EUSIPCO'04) (Vienna, 2004), pp. 1541-1548

20. F Tosato, P Bisaglia, Simplified soft-output demapper for binary interleaved COFDM with application to HIPERLAN/2, in IEEE International Conference on Communications, (ICC'02) (New York City, USA, 2002), pp. 664-668

21. STen Brink, J Speidel, RH Yan, Iterative demapping and decoding for multilevel modulation, in IEEE Global Telecommunications Conference (Sydney, NSW, 1998), pp. 579-584

22. NH Tran, HH Nguyen, Signal mappings of 8-ary constellations for bit interleaved coded modulation with iterative decoding. IEEE Trans. on Broadcasting. 52(1), 92-99 (2006)

doi:10.1186/1687-1499-2014-102

Cite this article as: Khan: Importance of noise models in FSO

communications. EURASIP Journal on Wireless Communications and

Networking 2014 2014:102. 